\title{
Developing a Group Model Building Causal Loop Diagram to Understand Millenial Generation Trust in Twitter as Early Warning System for Natural Disaster
}

\author{
Hilya Mudrika Arini ${ }^{*}$, Nurul Lathifah ${ }^{1}$, Fina Ananda ${ }^{1}$
}

\begin{abstract}
Nowadays, Twitter is used as an Early Warning System (EWS) for disasters because of the speed and many users. Based on Asosiasi Penyedia Jasa Internet Indonesia (APJII) data, in 2017, almost 50\% of internet users in Indonesia are born in 1983-1998. They are called the millennial generation. Therefore, this study aims to explore the trust of millennials towards Twitter as an EWS. This study utilizes the conceptual model from System Dynamics named Causal Loop Diagram (CLD) to identify the factors and the causal relationship among millennials' factors to trust Twitter as an EWS. It involves ten participants from the millennial generation, consisting of five passive Twitter users and five active Twitter users. A semi-structured interview was conducted with all participants to build the initial CLD gathered from each participant's perspective. Afterward, the initial CLD was verified by all participants through Focus Group Discussion. A group model building CLD that represents the influencing factors and their causal relationship of millennial generation trust in Twitter as EWS for a natural disaster is successfully developed from this study. The tweet frequency, the number of followers, the account credibility, the verified account, the level of trust in social media, and the content quality are considered the underlying factors of active and passive users to trust in Twitter as an EWS natural disaster.
\end{abstract}

Keywords: Early warning system, twitter, millennial, system dynamics, causal loop diagram, group model building.

\section{Introduction}

Indonesia is a country that regularly faces natural disasters, including tsunami and earthquakes. In 2018, there were 23 earthquake cases, and one tsunami case occurred, and more than ten million people were affected [1]. Many natural disaster casualties insist that the government find a proper early warning system (EWS). This system is supposed to minimize the number of casualties and ensure the residents can evacuate early and quickly. One of the EWS commonly used in Indonesia is inaTEWS (Indonesia Tsunami Early Warning System). The inaTEWS increases the awareness and prepa-redness of the residents in facing the potential and dangers of natural disasters. The improved awareness and preparedness can contribute to minimizing the number of casualties by providing information through electronic media, brochures, posters, and other media.

Internet is a medium for collecting accurate, complete, trustworthy, and relevant information [2]. However, nowadays, internet users had to face various problems, including boundless, inappropriate, inaccurate, and

'Faculty of Engineering, Department of Mechanical and Industrial Engineering, Universitas Gadjah Mada, Л. Grafika No 2, Kampus UGM, Yogyakarta 55281, Indonesia. Email: hilya.mudrika@ugm.ac.id

* Corresponding author irrelevant information. In addition, according to Asosiasi Penyedia Jasa Internet Indonesia (APJII) [3], in 2017 there were $54.68 \%$ of Indonesia's population using the internet. Based on these facts, the government took the initiative to expand inaTEWS using the internet as a medium of information. Social media is the chosen media to expand the inaTEWS; one actively used is Twitter. Twitter could provide more accurate and responsive information regarding early disasters warning [4]. This statement is supported by Chatfield and Bradjawidagda [4]. They analyzed Twitter for the earthquake in Bengkulu and Lampung in 2012. In this case, the government tweeted about the early warning of a tsunami, and four million Twitter users retweeted it within 15 minutes. This communication speed, reach, and information quality of early disasters warning affected by the active Twitter users in Indonesia. In addition, Carley et al. [5] also examined the use of Twitter in the 2014 earthquake in Padang. Carley et al. [5] examined the hashtags circulating on Twitter when the disaster issue occurred. They concluded the need for a comprehensive effort from Twitter as part of the early warning system so that the information can be disseminated quickly to the broader community. Thus, Twitter is a valuable media that could have a significant impact on early disasters warning.

APJII [3] classified internet users based on age. Almost $50 \%$ of internet users are people with the age range between 19-34 or people born in 1983 until 1998 
Table 1. Twitter users' characteristics

\begin{tabular}{llc}
\hline & Passive user & Active user \\
\hline Frequency of Twitter usage & Not open Twitter in the last 2 years & $\begin{array}{c}15 \text { minutes to } 2 \text { hours per day } \\
\text { Average }=1.03 \text { hours }\end{array}$ \\
& & Sd.Dev $=0.73$ hours \\
Last time of opening Twitter & More than 2 years ago & Less than 24 hours \\
Frequency of internet usage & 5 to 8 hours per day & 2 to 8 hours per day \\
& Average $=6.80$ hours & Average $=5.28$ hours \\
& Sd.Dev $=1.46$ hours & St.Dev $=2.65$ hours \\
\hline
\end{tabular}

[3]. This generation is known as the millennial generation [6]. This generation contributes to many internet usage percentages in Indonesia. Millennials are the first generation exposed to technology and feel comfortable using the internet [6]. Therefore, millennials tend to desire a quick, practical, and efficient approach to work. Millennials do not trust advertisements that provide one-way communication and information. However, they put high trust in information based on people's experiences. This characteristic increases the complexity in trust for using Twitter as the EWS. Therefore, a systematic thinking approach, mainly using the conceptual model of system dynamic named Causal Loop Diagram (CLD), is appropriately used to depict this causal relationship amongst factors in this problem.

Therefore, this study focused on exploring millennial trust in Twitter as an early warning system (EWS) in natural disasters by identifying underlying factors and their causal relationship using CLD. Additionally, this study attempted to distinguish Twitter's passive and active users regarding trust towards Twitter as EWS.

\section{Methods}

\section{Participants}

The chosen participant of this study complies with some criteria. The main criterium has a personal Twitter account. They should be a millennial generation born in 1983 until 1998, at the age range between 21-30 years and currently pursuing or having finished a bachelor's degree.

There are two groups of participants, the active and the passive Twitter users. A passive Twitter user refers to those who have a Twitter account, yet they are not active in tweeting and retweeting within two years. Meanwhile, an active Twitter user has a Twitter account and is actively tweeting, retweeting, and updating informa-tion on Twitter every day. Thus, in other words, the categorization for passive users and active users of Twitter depends on their activeness of using Twitter for accessing information, tweeting, and retweeting in a day, not based on their number of followers and following.
There were 10 participants involved in this study divided into five passive Twitter users ( $\mathrm{P} 1, \mathrm{P} 2, \mathrm{P} 3, \mathrm{P} 4$, $\mathrm{P} 5)$ and five active Twitter users (A1, A2, A3, A4, A5). Table 1 exhibits the characteristics differences between those two groups.

\section{Sample size}

This study is qualitative. The data collecting adopted saturation criterion to get a sufficient sample size $[7,8]$. A small number of samples or purposive samples was used and developed during the study and determined in advance. The interviewing was conducted until the participant's answers narrowed to a specific conclusion. In this study, ten participants participated.

\section{Data collection through interview description}

Generally, this study utilizes two data collection methods: semi-structured interview and Focus Group Discussion (FGD). First, all participants, both active and passive Twitter user, were interviewed individually in terms of (1) their trust in the use of Twitter as social media, (2) the factors that influence them to trust in Twitter, (3) their understanding of early warning system during a natural disaster, and (4) their trust and the factor to trust in the use of Twitter as an early warning system. Afterward, the interview results from each participant were summarized and represented in two initial Causal Loop Diagrams (CLDs), which consisted of initial CLDs for active and passive users. A semi-structured interview is a suitable method for developing CLD [9].

After two initial CLDs resulted from the semistructured interview, verification of CLDs was conducted using FGD. Five active users and five passive users participated in FGD separately. They discussed and revised the initial CLD whether the factors and the causal relationship amongst those in CLD fit with their perception. After the consensus was reached in each group, an empirical group model building CLD that can understand millennial generation trust in Twitter as EWS for natural disaster was obtained. 


\section{Results and Discussions}

\section{Trust in Twitter as a Social Media}

From the interview, passive users stated that they do not using Twitter frequently because they do not have many followers, and there is not much interesting news on Twitter (P2 and P5). P4 believed that the visualization of Twitter, which is text-based, makes it quite boring for the millennial generation. Using Twitter as a medium to gain information, passive users also rarely update news on Twitter. Besides, P4 stated that Twitter's main function is to find out information from their followed accounts, not the general updated information. This statement is different from P2 and P3, which says that updated information can be obtained from retweets generated and hashtags on Twitter. In general, passive users are more active in using other social media, such as Instagram, because the content is more attractive by showing images and videos ( $\mathrm{P} 4$ and $\mathrm{P} 5)$. However, the completeness of this feature will also affect the amount of storage required for the application. Both things will have an impact on user satisfaction. They also prefer other social media such as Instagram and Facebook because there is a limitation on the number of characters for a tweet, which only contain up to 280 characters. This limitation in the number of characters will cause misperception from readers. This misperception will result in a decrease in content quality (P4). Figure 1 shows the CLD of passive users' beliefs regarding Twitter's function as social media.

Besides, active users stated that they tend to use Twitter to find the latest information about the public interest. Four out of five active users (A2, A3, A4, A5) are already using Twitter for nine years. Active users have many followers and followings, ranging from 30 up to 400 friends. Like passive users, active users are also not updated on the news on Twitter because the information is obtained only from accounts that are followed (A4). Even though they are active in using Twitter, most participants (A1, A3, A4, A5) have higher user satisfaction when using social media such as Instagram due to better visualization and more friends than Twitter. Besides, A2 prefers using Twitter because the application data quota used when using this application is lower than using Instagram. Higher user satisfaction will increase higher user trust in social media. The frequency of social media usage also influences the level of trust in social media. The more often they use certain social media, the more users will trust them. Figure 2 shows the CLD generated from interviews with active users on Twitter's role as social media.

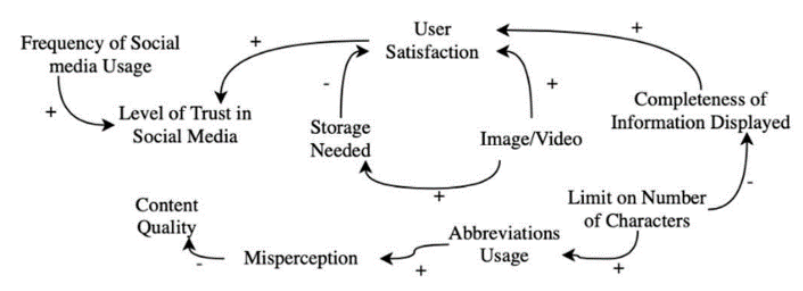

Figure 1. Trust of passive users in Twitter as social media

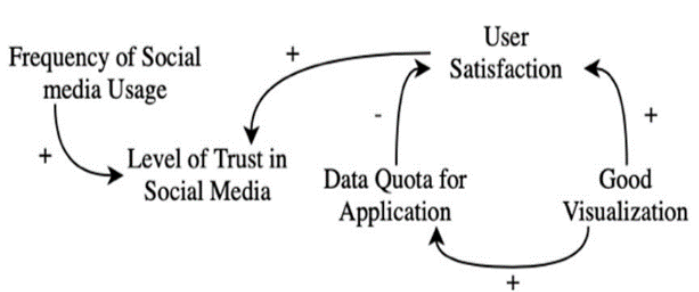

Figure 2. Trust of active users in Twitter as social media

trust because the probability of hoax information is lower than the un blue checkmark account. Apart from verified accounts, another factor that affects their trust in accounts on Twitter is the number of followers of the account. P1, P2, and P3 emphasized that the number of followers can indeed be used as a reference because it indicates that the information associated with these accounts is accurate. Another factor that was considered is content quality. P2 said that when the tweets are full of information, the number of retweets is high, and followers' comments will not be a major consideration. Content quality will increase the number of retweets. The number of retweets will expand the tweet's reach and increase the speed of updating news on Twitter.

The number of tweets has not associated with the account to be followed since the number of tweets depends on how long the user active on Twitter. Tweet frequency is also important. A higher tweet frequency will increase a higher trust because it will assume that the account is responsive to news (P5). $\mathrm{P} 2$ and $\mathrm{P} 4$ believe that mutual friends will not affect someone's trust in an account on Twitter. They think someone will follow an account due to the readers' perception and interest in the account.

On the other hand, P1, P3, and P5 stated that the number of mutual friends is one of their considerations when choosing an account to follow. P2 and P5 also said that the image of the account owner could also influence the decision to follow an account. The image of the account owner can be seen from the display picture and biography used (P5). In addition, the number of positive comments on their tweets reflects the image of the account owners. 


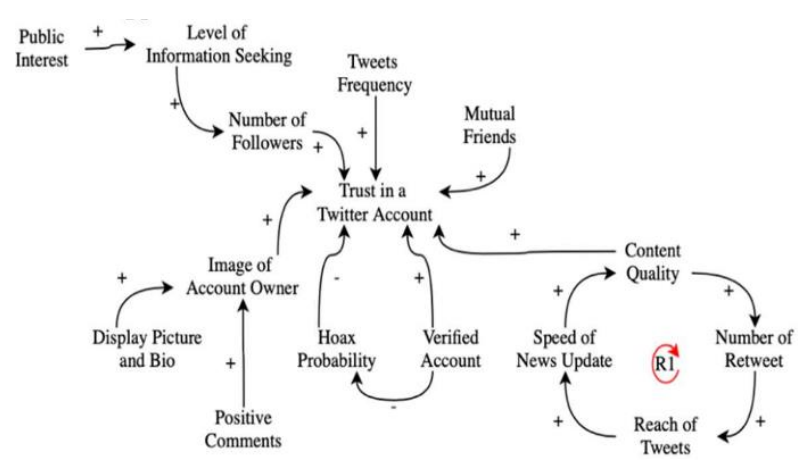

Figure 3. Trust of passive users in factors affecting trust in Twitter.

Figure 3 shows CLD, which illustrates passive user trust in the factors that support trust in a Twitter account.

Meanwhile, four out of five participants (A2, A3, A4, A5) of active users thought that content quality is the main thing to be considered in following an account. Content quality can be seen from the information displayed and also the way it is delivered. Good content quality is indicated by many retweets and can be seen from how quickly the account updates about the latest information. Good content quality is one of the initial considerations for building trust (A5). Another factor to take into consideration is the number of followers. The number of followers of an account indicates the number of Twitter users who trust and are interested in the account, so this factor must be considered (A1 and A5). The number of followers also shows the credibility of the account. The larger the number of followers, the higher is the credibility of an account. However, many followers must be considered by the absence of negative comments on the account (A4).
A verified account is also one of the considerations in following an account (A1, A3, A5). According to A3, a verified account is unlikely to provide hoax information. Even though the probability of hoax information is very small, misperceptions are still possible because of the character limitations on Twitter, particularly for people who are not common in using Twitter (A3). Apart from character limitations, misperceptions may also occur due to differences in understanding readers from various backgrounds (A2). Information accuracy is also an important factor since the more accurate the information generated by an account, the greater the user's trust in that account. Trust in the organization is also important to increase trust in an account (A3). Vice versa, trust in an account will increase a person's trust in an organization. Like passive users, four out of five active users (A2, A3, A4, A5) thought that the number of tweets does not need to be considered in following an account. Meanwhile, A1 believes that the number of tweets is important to be considered because the number of tweets will indicate the level of activity of an account. CLD, which illustrates factors that influence active users' trust in a Twitter account, is shown in Figure 4.

\section{Understanding of Early Warning System}

According to the passive users, EWS is important and useful for reducing the risks of disasters. The existence of EWS will increase people's disaster awareness and assist in the decision-making process in evacuation preparedness (P2). However, EWS cannot be used for unpredictable disasters such as earthquakes but has a significant impact on disasters, including tsunamis and volcanic eruptions, because early signs appear before the disasters occur (P5). Four out of five participants (P2, P3, P4, and P5)

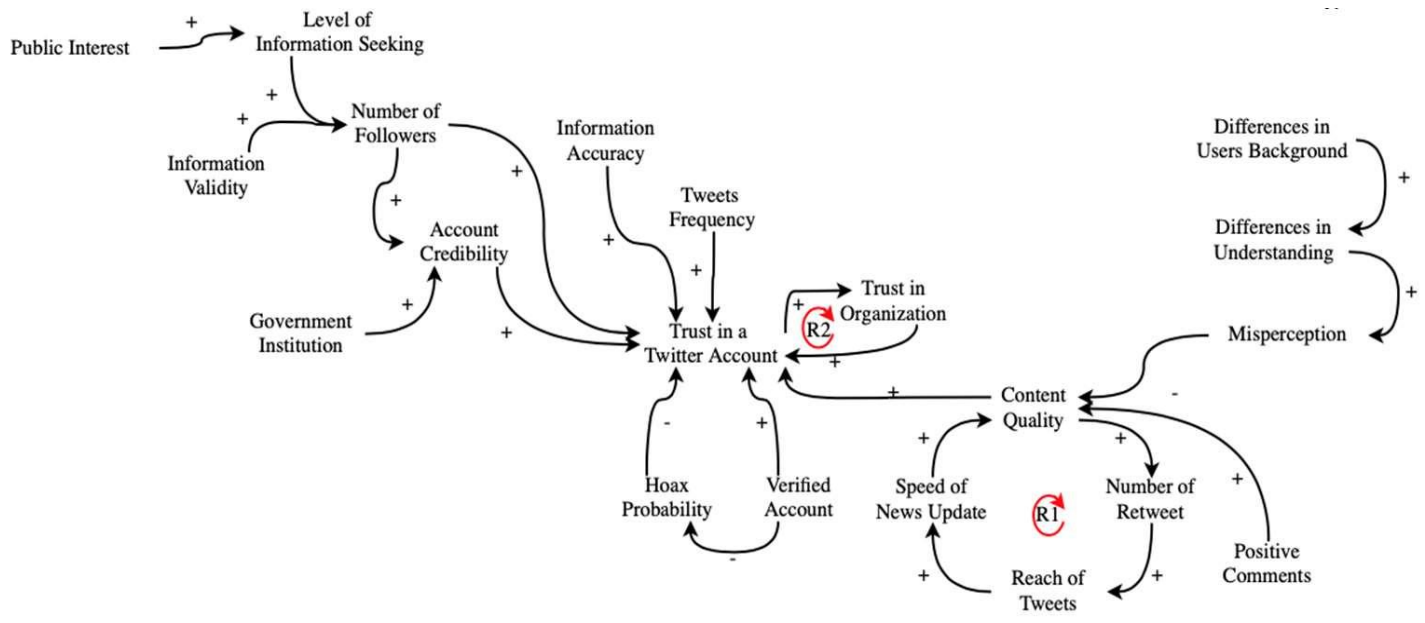

Figure 4. Trust of active users in factors affecting trust in Twitter. 


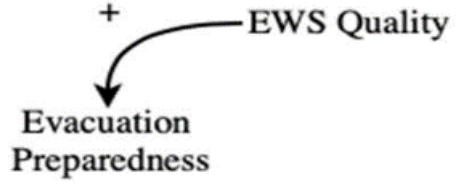

Figure 5. Trust of passive users in EWS

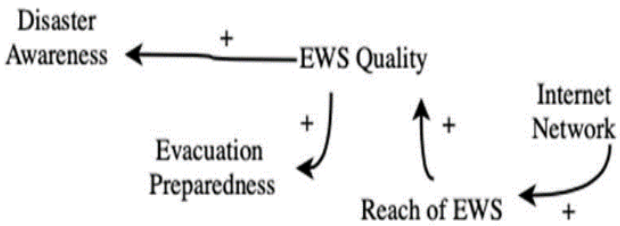

Figure 6. Trust of active users in EWS

stated that they usually obtained EWS-related information through chat applications, such as WhatsApp and Line. At the same time, the remaining participant $(\mathrm{P} 1)$ stated that information about the EWS is obtained via Instagram. All participants agreed that the information obtained is usually a quote from Twitter. Figure 5 shows the CLD showing millennial trust in the EWS.

Besides, participants from active users stated that EWS is important and useful for providing warnings to the residents and communities regarding upcoming disasters. The existence of an EWS will increase disaster awareness and assist in the decisionmaking process in preparation for evacuation (A2). However, the online-based EWS application does not have a significant effect because some areas have not been covered yet by the internet (A5). Three out of five active Twitter users (A3, A4, A5) used Twitter to identify potential disasters through the EWS. The information obtained from BMKG or information retweeted by the accounts that they followed. In addition, the participants also trusted chat applications such as Line and Whatsapp, television, and the BMKG official application. Television has a wider reach than the internet because electricity is easier to find than the internet network. CLD, which illustrates millennial trust in EWS, is shown in Figure 6.

\section{Trust in Early Warning System on Twitter}

The EWS is currently carried out through social media to reach a wider audience [5]. However, this benefit is limited to people using the internet in their daily lives (P5). The main problem with the deployment of EWS is its coverage which depends on the internet network (P5). The internet network will also affect EWS accessibility. In terms of the speed of updating news about disasters, a participant stated that Meteorological, Climatological, and Geophysical Agency's (BMKG) Twitter account is the most responsive account in spreading news. All passive

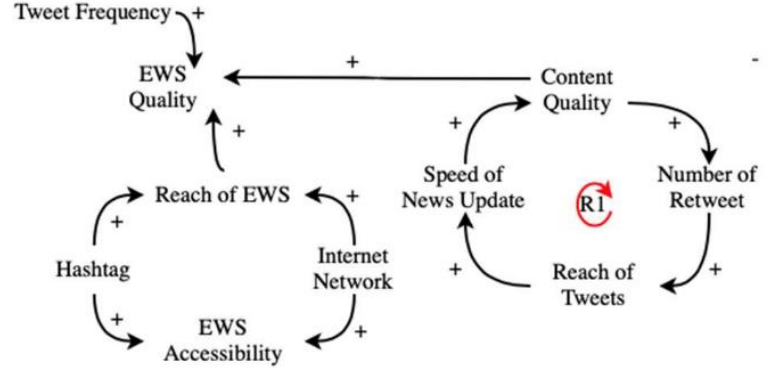

Figure 7. Trust of passive users in EWS on Twitter

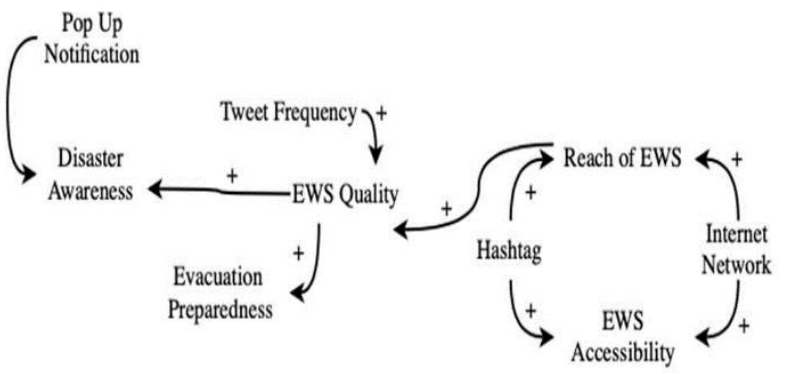

Figure 8. Trust of active users in EWS on Twitter

participants trusted EWS and other information related to disaster in Indonesia from the BMKG official account. P1 and P2 chose BMKG because this account is verified and is a government institution.

Meanwhile, P3 chose BMKG because the average friends they followed on Twitter also followed the BMKG account. This account is also trusted because the information update is faster than other accounts, and there is information about the weather condition every day (P5). According to participants, the EWS on Twitter is effective in predicting disasters (P5). The use of Twitter is more effective because of the retweet feature that can expand the reach of news or information and hashtags to find out the latest trending topics (P3). EWS on Twitter is more effective than EWS on television because Twitter content is text-based and does not require visual content (P3 and P4). Figure 7 shows the CLD depicting millennial trust in EWS on Twitter.

Active users stated that they are sufficiently updated on EWS information on Twitter. However, they considered that giving an EWS via Twitter is not very effective. Two out of five participants (P1 and P2) prefer to use the BMKG application instead of opening Twitter to gain information about the disaster because Twitter's main function is entertainment. A participant said that if we want to find out information related to the EWS, which BMKG usually writes, it is better to immediately open the BMKG application (A1). The information that appears on Twitter is very boundless, so the probability of BMKG information not being read by Twitter users is very high (A2). However, according to A4, Twitter is a suitable medium for spreading information because of 


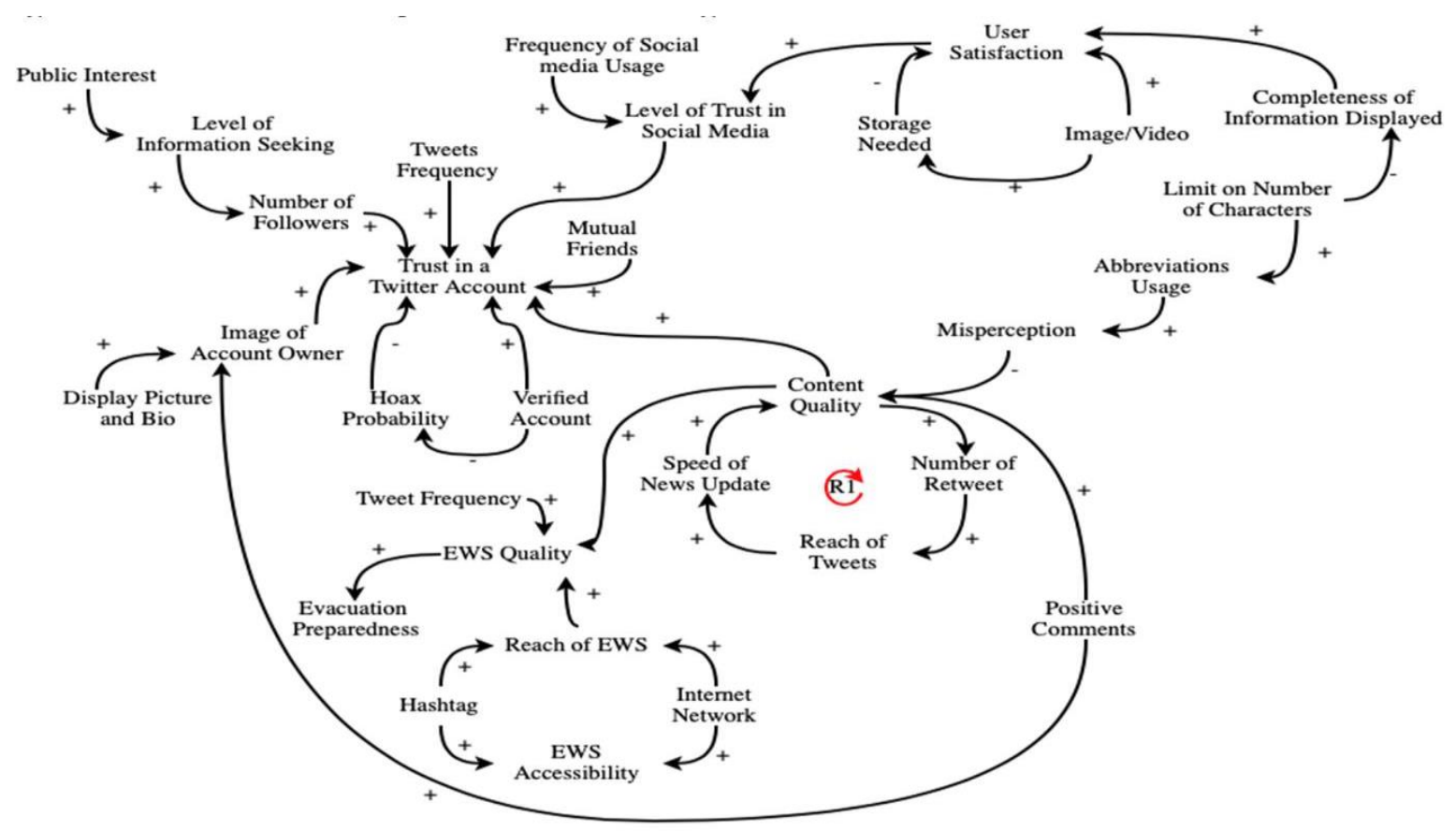

Figure 9. Initial CLD for passive users

the retweet feature and text-based information. A3 claimed that Twitter as EWS could be optimized if it is equipped with a pop-up notification feature. All participants trusted the BMKG account in spreading EWS news. One of the considerations is many followers (A1) and the institution that has the authority to spread the news. In terms of speed, BMKG's Twitter account provides the most updated information compared to other platforms. However, the speed of spreading information also depends on the internet network in disaster conditions (A2). Figure 8 is a CLD depicting active Twitter users' trust in EWS on Twitter.

\section{Initial causal loop}

Based on the interview results, we construct the initial CLD. The interviews are transcript using NVivo 12. The analysis was conducted separately between passive and active users of Twitter. All discussed results are summarized as the initial CLD, as shown in Figure 9.

Initial CLD for passive users shows that there is one reinforcing loop (R1). R1 loop consists of 4 factors: The content quality will affect the number of retweets, the number of retweets will increase the reach of tweets, and the reach of tweets will increase the speed of news updates. Besides, three factors influence each other. There is trust in a Twitter account, verified account, and hoax probability. A verified account will reduce the hoaxes probability and will increase the trust in a Twitter account. This finding is supported by Thomson et al. [10] that said tweets from government official verified accounts would be retweet more often, and the information spreads will be wider than tweets from non-verified users' accounts. From the CLD can be seen that trust in a Twitter account is positively influenced by the number of followers, image of the account owner, verified account, mutual friends, level of trust in social media, and tweets frequency. A positive sign means strengthening, which means that if the influencing factors increase, then the trust in a Twitter account will also be increased.

Meanwhile, trust in a Twitter account is also negatively affected by the hoax probability. This means that if the hoax probability increases, the level of trust in a Twitter account will be decreased. Rumors or misinformation will reduce user trust and increase chaos in a situation [11].

The account owner's image is influenced by the display picture and bio of the account and the number of positive comments in each tweet. Besides, the number of followers will be influenced by the level of information searching. The higher the level of information searching, the number of followers of an account associated with that information will also be increased. The level of information searching is also positively influenced by the public interest. Comrie et al. [12] stated that if information becomes a public interest, many people will look for the information from Twitter. Therefore, the public interest will increase the level of information searching. In 


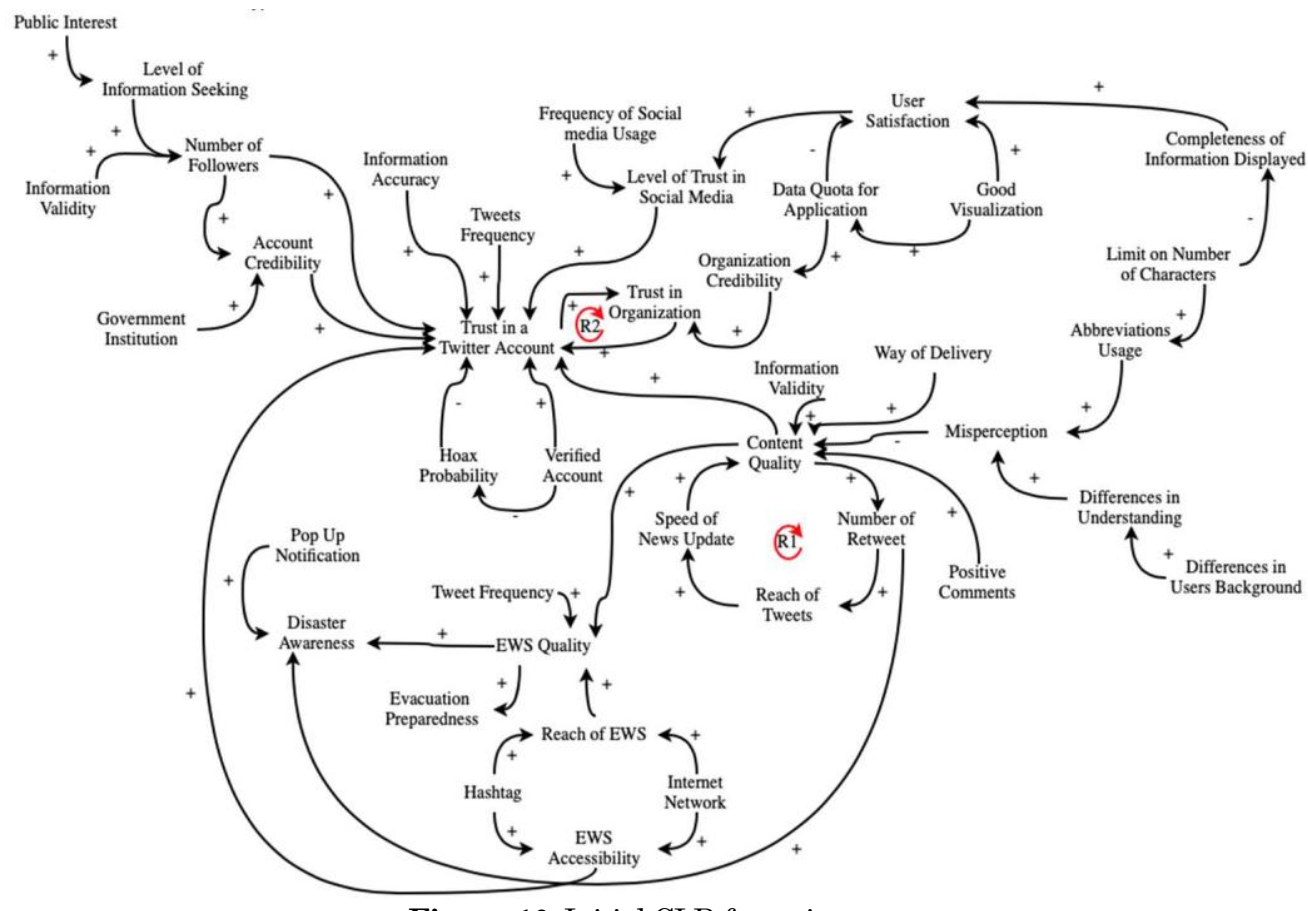

Figure 10. Initial CLD for active users

addition, the level of trust in social media is influenced by the frequency of social media usage, where the more often the social media being used, the more users will trust the social media. The CLD also shows that the EWS quality is influenced by two factors: the reach of EWS and the tweets frequencies. Both are mutually reinforcing (marked with a positive sign). The EWS quality on Twitter will affect evacuation preparedness [13]. The higher the EWS quality, the better evacuation preparations can be made. The reach of EWS forms a causal relationship with other factors such as the internet network, hashtags, and EWS accessibility. Thus, having hashtags will increase the reach of EWS as well as EWS accesssibility. The internet network shows real-time information so that risk can be reduced [14]. Therefore, the use of the internet as EWS will increase the reach and accessibility of the EWS.

From the CLD can be seen that content quality is negatively affected by misperception. Even though Twitter usage effectively spread information, Twitter can be difficult to use in a disaster context. For instance, in Twitter, there can be inaccurate tweets [10], and false information [15] exist, which will cause misperception to users [5]. The greater the misperception appears, the worse the content quality of an account will be. In addition, the content quality is also affected by the speed of news updates. The earlier the news appears, the better the quality of the content. The content quality also affects the number of retweets. The better the content quality on Twitter, the more accounts will retweet the tweet. The relationship between content quality and the number of retweets is in line with research by Bornmann and
Haunschild [16], which stated that a large number of retweets is one aspect that must be seen to assess the quality of tweets. In addition, due to the limitation of 280 characters, abbreviations trigger misperception in a tweet. Character limitation is a Twitter characteristic [17]; thus, it decreases the level of completeness of the information that can be displayed. Whereas in fact, completeness of information will increase user satisfaction. User satisfaction is the most important thing to consider in developing an information system or application [17]. User satisfaction also forms an opposite relationship with other variables, including the storage space needed for applications and images or videos that make the visualization more attractive. Good visualization will certainly increase user satisfaction, but applications that display good visuals will also take up more space on a smartphone.

On the other hand, a lot of application storage space will certainly reduce user satisfaction. Apart from affecting the image of the account owner, positive comments also affect the content quality of an account on Twitter. The more positive comments on an account will show that the account has good content quality in the perceptions of other Twitter users. The initial CLD for Twitter active users can be seen in Figure 10.

Initial CLD for active users shows two reinforcing loops. Four factors constructed R1 loop: content quality, number of retweets, reach of tweets, and speed of news update. While in R2, there are only two factors: trust in a Twitter account and trust in an organization. CLD shows, trust in a Twitter account 


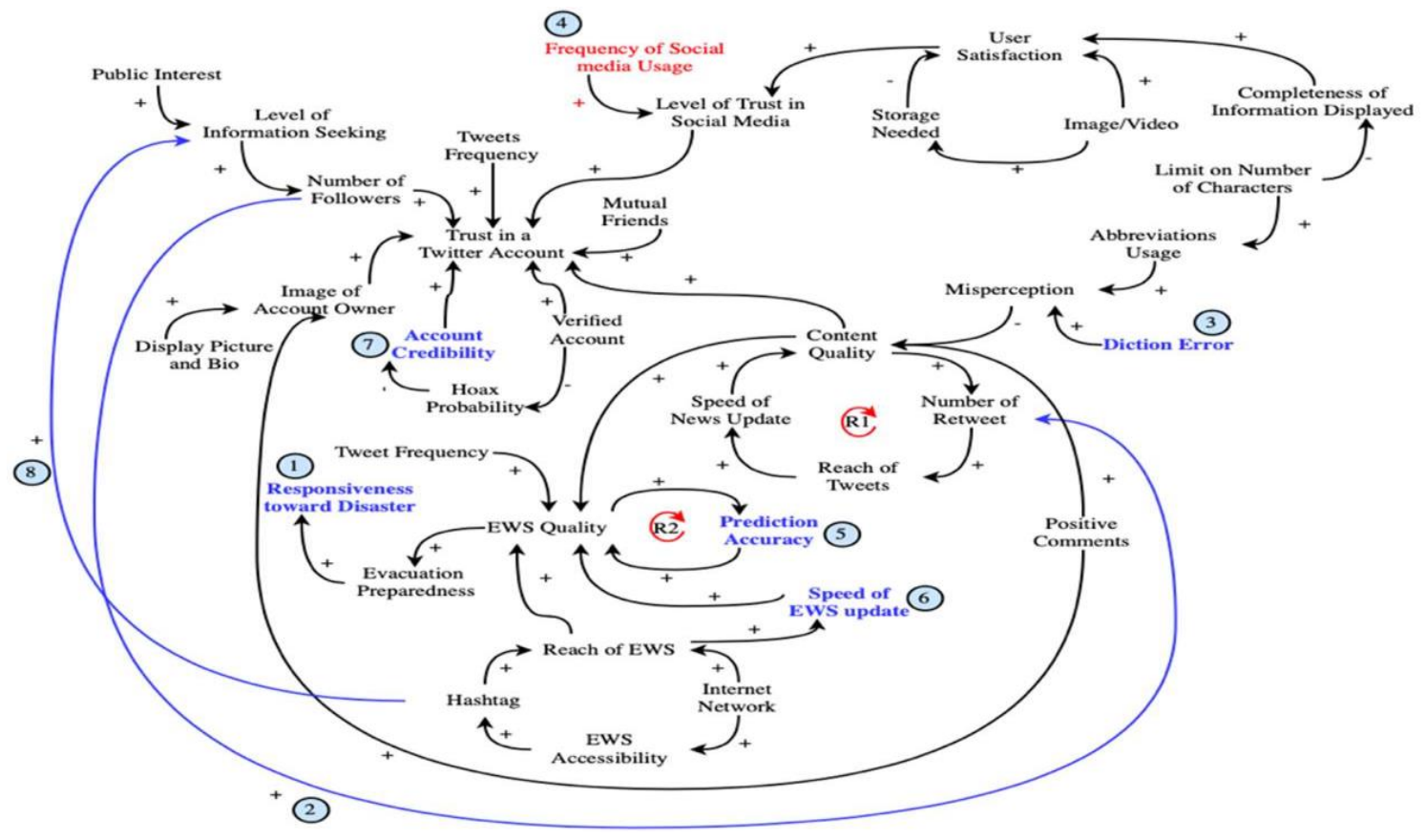

Figure 11. Verified CLD for passive users.

is positively influenced by the number of followers, account credibility, verified account, hoax probability, information accuracy, tweets frequency, level of trust in social media, and trust in an organization. A positive sign means strengthening in the loop, which means that if the influencing factors increase, then the trust in a Twitter account will also be increased. The account popularity was influenced by the number of tweets post in a certain period and the number of followers [16]. However, trust in a Twitter account is negatively affected by hoax probability which means that if hoax probability increases, the level of trust in a Twitter account will be decreased. This finding is in line with Castillo et al. [11], which stated that misinformation would decrease user trust and increase chaos in a situation.

In contrast with the initial CLD for passive users, that considered the image of account owner would influence users' trust. In this CLD, active users agreed that the account credibility influences users' trust. Participant A1 and A2 agreed that government institution accounts are usually more credible than other accounts. Tweet from governments and other official accounts are trustworthy [10]. News accuracy and trust in an organization are also important in choosing whether to follow the account.

Like the finding for passive users, this CLD shows that the level of information searching will influence the number of followers. The higher the level of information searching, the number of followers related to that information will also be increased. The level of information searching is also positively influenced by the public interest. If information becomes public interest, many followers will seek that information from Twitter. Thus, the public interest will increase the level of information searching [12]. In addition, the level of trust in social media is influenced by the frequency of social media usage; when the frequency of social media usage is higher, the users will put more trust in social media. From the CLD can be seen that EWS quality is influenced by two factors, that are reach of EWS and tweet frequency. EWS quality on Twitter will affect evacuation preparedness and disaster awareness of users [5]. The higher the EWS quality, the evacuation preparation will be better [13]. The reach of EWS is influenced by other factors, including the internet network, hashtags, and EWS accessibility. Social media is generally considered a "quick way to communicate" [5]. Thus, warnings spread by media, such as Twitter, will have a wider reach. A positive sign indicates that a hashtag will increase the reach of EWS, the EWS accessibility, and the internet network. Apart from the positive impact, hashtags on Twitter also have negative impacts. The hashtags do not contain spaces, and capital letters trigger confusion [5]. Flanagan [14] stated that the internet network would help provide real-time information, leading to decreasing risk.

CLD shows that content quality is negatively influenced by misperceptions which means that if the misperceptions exist, the content quality of an account will be worse. The way of delivery and information validity are also the factors that affect content quality positively. The better the way of delivery and the higher information validity, the content quality will be better. Moreover, the content 


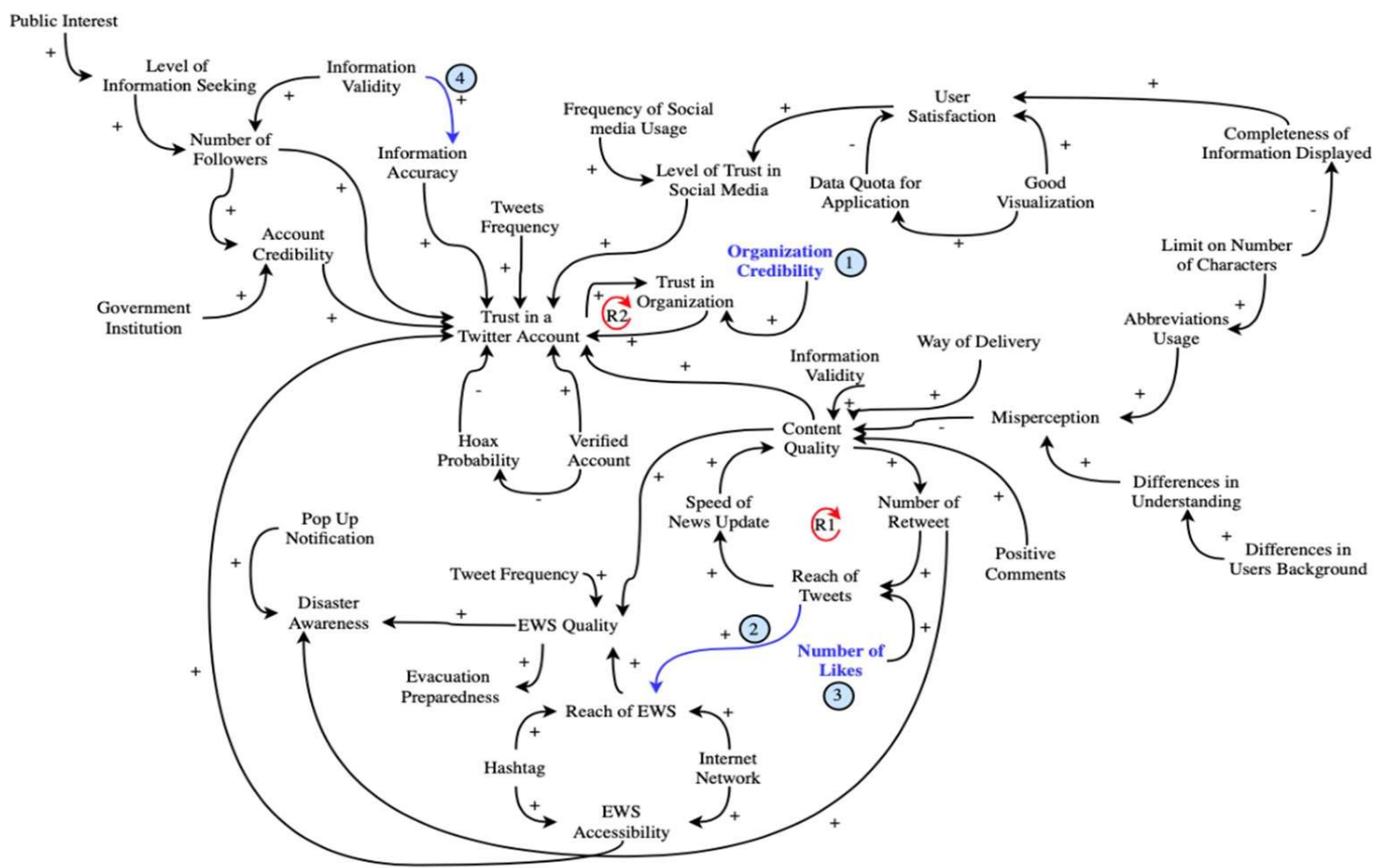

Figure 12. Verified CLD for active users.

quality is also affected by the speed of news updates. The sooner the news appears, the content quality will be better. The content quality also affects the number of retweets. The content quality will determine the number of retweets at the initial tweet [16]. The relationship is that the better the content quality on Twitter, the more accounts retweet the tweet. These four factors made up the loop R1. Apart from that, positive comments also affect the content quality. The more positive comments appear, the better a tweet is from the Twitter users' perspective. Active users said that the misperception was caused by the abbreviations usage, which resulted from the limitation for characters in one tweet. The limitation on the number of characters which is a characteristic of Twitter, will reduce the level of completeness of the information displayed [17]. Whereas in fact, completeness of information will increase user satisfaction. According to Liu et al. [17], user satisfaction is the most important factor to consider in building an information system. User satisfaction also forms a loop with other variables: data quota for application and good visualization. Good visualization will certainly increase user satisfaction, but visualization will also use up many data quotas. On the other hand, a lot of data quota for applications will certainly reduce user satisfaction. Apart from the above factors, another factor that also influences misperception is the difference in understanding caused by the different backgrounds of Twitter users.

\section{CLD verification using focus group discussion}

Verification was conducted to synchronize the perceptions among participants in this study. The verification process was carried out using the focus group discussion (FGD) method by showing the initial CLD to the participants and searching their initial CLD. The response could be by adding new nodes, deleting nodes, adding links, and others. FGD was conducted separately between passive and active users of Twitter. The verification through FGD was carried out in two iterations. The first iteration was based on individual opinion, while the second iteration was based on the final discussion with the group. Figure 11 shows the updated CLD of passive users verified through FGD.

Some changes occurred in CLD for passive users. There was an addition of "Responsiveness toward Disaster" because of evacuation preparedness. Having a good evacuation preparation will affect the responsiveness of people toward disaster (P2). Twitter provides EWS to deliver updated information and suitable evacuation routes and planning to respond to the disasters that exist [18]. In addition, a link was added between the number of followers and the number of retweets. Many followers indicates that many people trust the information from an account. Having more followers means that the probability of more people seeing a tweet is high, increasing the number of retweets (P4). The number of retweets on 
an account can describe the level of account popularity [16].

Moreover, the "speed of EWS update" factor was also added to improve the EWS quality and be influenced by the reach of EWS (P1). These three factors are associated with a positive sign (+). Another change is adding an "Account Credibility" factor between the hoax probability factor and trust in a Twitter account factor. The addition was made because hoaxes do not directly affect a person's trust (P1). Tweets from the government or verified officials' accounts are credible in spreading information [10]. The last change is to make a relation between hashtags and the level of information seeking. Hashtags are used to search some information by combining several keywords or phrases on Twitter. Thus, the presence of hashtags will increase the search result for related information (P4). Figure 12 shows the updated CLD of active users verified through FGD.

Some changes were conducted to CLD of active users of Twitter. Changes that occurred were by adding the "Organization Credibility" factor to support the trust in the organization factor. The public will easily trust an organization if the organization is credible and can generate positive news and information for the public (A1). In addition, the reach of tweets will affect the reach of EWS. It might happen because the wider tweets spread and the more people read tweets containing information about EWS, the reach of EWS will be expanded by using a retweet feature on Twitter (A5).

Another change was made by adding the 'Number of Likes' factor to support the reach of tweets. Activities of Twitter users, including replies, retweets, and likes, will appear on the timeline so that the number of likes must also be included to support the reach of tweets factor (A3). In addition, there was a link added between the information validity and the information accuracy factor. Valid information means more accurate information that spread. Thus, validity will affect the accuracy of the information received (A1).

\section{Conclusions}

This paper presented CLD constructed based on the similarities and differences of passive and active Twitter users' responses. According to passive users, they trust in a Twitter account as EWS because of some factors, i.e., the number of followers, the tweets frequency, the image of the account owner, the account credentials, the verified account, the mutual friend, the level of trust in social media and the content quality. Moreover, instead of EWSupdate speed, the prediction accuracy, and the reach of EWS, the tweet frequency and the content quality also influence EWS quality that subsequently it will indirectly affect their trust in Twitter as EWS.

In contrast with the passive user, the active users have more factors. The tweet frequency, the number of followers, the account credibility, the verified account, the level of trust in social media, and the content quality are similar factors between active and passive users to trust in a Twitter as EWS. Meanwhile, the information accuracy, the trust in the organization, the hoax probability, and the EWS accessibility become some distinct factors between active and passive users to trust in Twitter as EWS. However, relatively like passive users, EWS quality is also affected by tweet frequency, the reach of EWS, and content quality.

This study concluded to raise millennial generation trust, some factors should be considered by the government institution and non-government organization who desire to use or improve twitter performance as EWS when a natural disaster occurs. They should increase their Twitter account credibility by adding the frequency of tweets and the number of followers, improving the image of the account owner, converting to a verified account, and enhancing the content quality. Particularly for millennials who are active Twitter users, the content quality can be increased by minimizing hoax probability and maximizing the information accuracy. This step is crucial because the content quality is highly correlated to EWS quality. Additionally, from their perception, government should provide the ease of access to internet networks during the disaster if they intend to use Twitter as EWS for a further natural disaster.

\section{References}

1. Badan Nasional Penanggulangan Bencana (BNPB), Bencana Tahun 2018, 2018, retrieved from https://apjui.or.id/.

2. Klein, B. D., User Perceptions of Data Quality: Internet and Traditional Text Sources, Journal of Computer Information Systems, 41(4), 2001, pp. 9-14.

3. Asosiasi Penyelenggara Jasa Internet Indonesia (APJII), Penetrasi dan Perilaku Pengguna Internet Indonesia 2017，2017, retrieved from https://apjii.or.id/.

4. Chatfield, A. T., and Brajawidagda, U., Twitter Early Tsunami Warning System: A Case Study in Indonesia's Natural Disaster Management, Proceedings of the Annual Hawaii International Conference on System Sciences, 2013, pp. 2050 2060.

5. Carley, K. M., Malik, M., Landwehr, P. M., Pfeffer, J., and Kowalchuck, M., Crowd Sourcing Disaster Management: The Complex 
Nature of Twitter Usage in Padang Indonesia, Safety Science, 90, 2016, pp. 48-61.

6. Strauss, W., and Howe, N., Millenials Rising: The Next Great Generations, Vintage Books, New York, 2000.

7. Mason, M., Sample Size and Saturation in $\mathrm{PhD}$ Studies Using Qualitative Interviews, Forum Qualitative Social Research, 11(3), 2010.

8. B Saunder, B., Sim, J., Kingstone, T., Baker, S., Waterfield, J., Bartlam, B., Burroughs, H., and Jinks, C., Saturation in Qualitative Research: Exploring Its Conceptualization and Operationalization, Quality and Quantity, 52(4), 2018, pp. 1893-1907.

9. Sterman, J. D., Business Dynamics: Systems Thinking and Modeling for a Complex World, McGraw-Hill, Boston, 2000.

10. Thomson, R., Ito, N., Suda, H., Lin, F., Liu, Y., and Hayasaka, R., Trusting Tweets: The Fukushima Disaster and Information Source Credibility on Twitter, 9th ISCRAM Conference, 2012, p. 10.

11. Castillo, C., Mendoza, M., and Poblete, B., Information Credibility on Twitter, Proceedings of the 20th International Conference on World Wide Web, 2011, pp. 675-684.

12. Comrie, E., Burns, C., Coulson, A., Quigley, J., and Quigley, K., Rationalising the Use of Twitter by Official Organisations During Risk Events:
Operationalising the Social Amplification of Risk Framework through Causal Loop Diagrams, European Journal of Operational Research, 272(2), 2019, pp. 792-801.

13. Santos, R. M., Mosse, D., Znati, T., and Comfort, L. K., Design and Implementation of a Witness Unit for Opportunistic Routing in Tsunami Alert Scenarios, Safety Science, 90, 2016, pp. 75-83.

14. Flanagan, J., Early Warning System for Natural and Manmade Disasters, US Patent 1999.

15. Tinker, T., and Vaughan, E., Risk and Crisis Communications: Best Practices for Government Agencies and Non-Profit Organizations, Booz Allen Hamilton, 2010.

16. Bornmann, L., and Haunschild, R., T factor: A Metric for Measuring Impact on Twitter, Malaysian Journal of Library and Information Science, 21(2), 2016, pp. 13-20.

17. Liu, I. L. B., Cheung, C. M. K., and Lee, M. K. O., Understanding Twitter Usage: What Drives People Continue to Tweet, Proceedings of the Pacific Asia Conference on Information Systems, 92, 2010, pp. 927-939.

18. Landwehr, P., Wei, W., Kowalchuck, M., and Carley, K. M., Using Tweets to Support Disaster Planning, Warning and Response, Safety Science, 90, 2016, pp. 33-47. 